\title{
Adaptive pulse width modulation design for power converters based on affine switched systems
}

\author{
Simone Baldi ${ }^{\mathrm{a}, *}$, Antonis Papachristodoulou ${ }^{\mathrm{b}}$, Elias B. Kosmatopoulos ${ }^{\mathrm{c}}$ \\ ${ }^{a}$ Delft Center for Systems and Control, Delft University of Technology, Delft 2628CD, The Netherlands \\ ${ }^{b}$ Department of Engineering Science, Control Group, University of Oxford, Parks Road, Oxford OX1 3PJ, U.K. \\ ${ }^{c}$ Dept. of Electrical and Computer Engineering, Democritus University of Thrace, Xanthi 67100, Greece and \\ Informatics \& Telematics Institute, Center for Research and Technology Hellas (ITI-CERTH), Thessaloniki 57001, Greece
}

\section{Abstract}

In this work we propose a novel adaptive switching strategy for the design of pulse width modulation signals in power converters. Instead of an uncertain averaged model of the power converter, an uncertain switched model is considered, which can better represent the actual power converter dynamics. Uncertainties in the power converters parameters are handled via an adaptive control approach, and all circuit parameters of the switched model are assumed to be unknown (including the load and parasitic effects). After defining the pulse width modulation in terms of a reverse mode-dependent dwell time, an elementarytime-unit Lyapunov function is used to derive a set of linear matrix inequalities (LMIs) based on global uniformly ultimately boundedness of the switched system. The LMIs are solved in an adaptive fashion using an exploitation-exploration mechanism: exploitation is achieved by solving the LMIs based on the estimated switched model, while exploration is achieved by a persistently exciting input voltage source, which guarantees convergence of the estimated parameters to the true system parameters.

Keywords: Adaptive Control, Switched Systems, Mode-dependent Dwell Time, Power Converters

\section{Introduction}

Switching-mode devices are crucial in many applications in industrial and power electronics. The use of pulse width modulation (PWM) signals to drive the switching behavior is the key in the conversion between direct current voltages in DC-DC power converters. In such devices, the switching PWM signal has to be generated to regulate an output voltage, despite of changes in the load [1,2]. Different control designs to generate the PWM consider an averaged model for the converter [3]. This modeling approach averages the switching dynamics over the period of the pulse signal. The main advantage of averaged dynamics is their suitability for existing control designs, e.g. power converters with bilinear averaged dynamics have been analyzed by using Hamiltonian methods [4]. If the PWM signal is of sufficiently high frequency, the behavior of the averaged system will be close to the behavior of the original switching-mode converter. However, in case some of the assumptions for the averaged model do not hold, e.g. if the PWM is not of high frequency, a hybrid (switched) model for the converter is preferred and the development of a control design strategy for this switched model is of utmost importance [5].

\footnotetext{
${ }^{*}$ Corresponding author. Tel.: +31152781823

Email addresses: s.baldi@tudelft.nl (Simone Baldi), antoniseeng.ox.ac.uk (Antonis Papachristodoulou), kosmatop@iti.gr (Elias B. Kosmatopoulos)
} 
As an example, let us focus on the boost converter, whose averaged and switched models are described in Appendix A with parameters as in Section VI. To explain the mechanism through which a desired voltage is achieved, we illustrate the behavior arising by switching from one mode to the other and compare it with the averaged model behavior. Figs. 1(a) and 1(b) show the phase plane of the two modes of the boost converter (mode 1 and 0 , with closed and open switch respectively). Both modes have a stable equilibrium, with the first mode having two real eigenvalues, and the second mode having a pair of complex conjugate eigenvalues. When the two modes are combined via the duty cycle, the trajectories switch from the first to the second mode and vice versa. The resulting dynamics can be represented by the switched system

$$
\dot{x}(t)=A_{\sigma(t)} x(t)+E_{\sigma(t)} v_{s}, \quad \sigma(t) \in\{1,0\},
$$

where the signal $\sigma(t)$ is a time-dependent signal that determines at every time the switching between closed $(\sigma(t)=1)$ and open switch $(\sigma(t)=0)$; the matrices $A_{\sigma}$ and $E_{\sigma}$ contain the parameters of the boost converter operating in mode $\sigma$ (cf. Appendix A); the state $x=\left[i_{L} v_{\text {out }}\right]^{\prime}$ is a bidimensional vector composed of the inductor current $i_{L}$ and output voltage $v_{\text {out }}$; and $v_{s}$ is the (typically constant) input voltage.

Switching with a certain duty cycle $D$ between the two modes makes the trajectories converge to a limit cycle, as shown in Figs. 1(c) and 1(d), which have been obtained for $v_{s}=12 \mathrm{~V}$ and $D=0.5$. On the other hand, according to the averaged model of the boost converter (see its derivation in (A.3)), instead

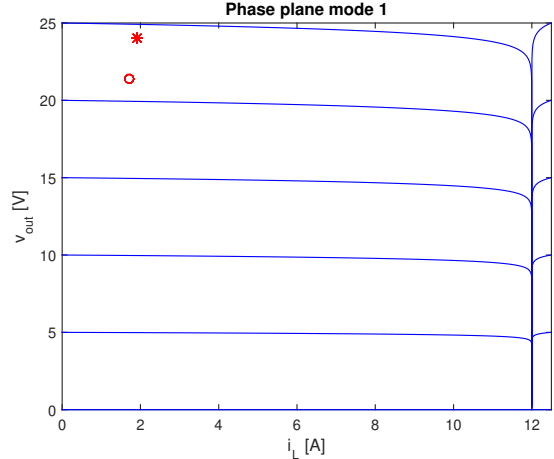

(a) Mode 1

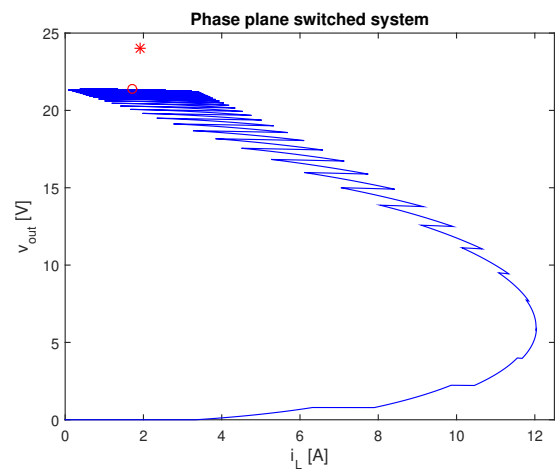

(c) Hybrid behavior

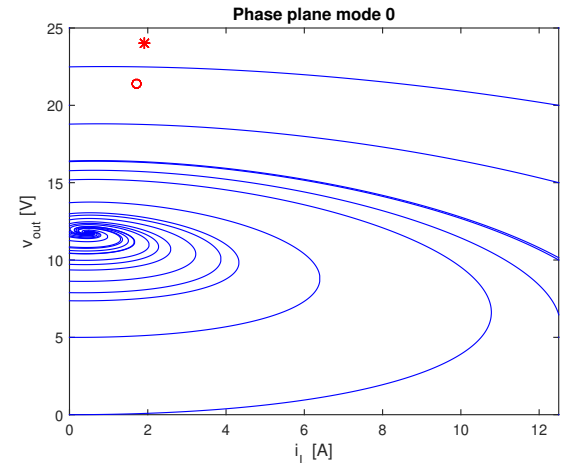

(b) Mode 0

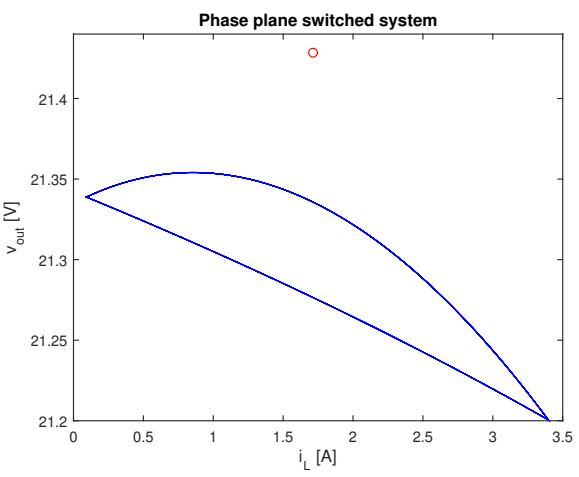

(d) Detail of the limit cycle

Figure 1: Phase plane of the two stand-alone modes, and of the switched model with duty cycle $D=0.5$ (the ideal state is indicated with a star, and the equilibrium of the averaged model with a circle) 
of converging to a limit cycle, the trajectories will converge to an isolated fixed point, indicated with a circle in Figs. 1(c) and 1(d). Since $v_{s}=12 \mathrm{~V}$ and $D=0.5$, ideally, one would expect to cycle around $v_{\text {out }}$ des $=v_{s} /(1-D)=24 \mathrm{~V}$. However, a first crucial observation is that, due to non-ideal effects like parasitic losses, the limit cycle obtained with the ideal duty cycle can be far from the desired output voltage (indicated with a star in Fig. 1(c)). Figs. 1(c) and 1(d) have been obtained with a switching period of $65 \mu$ s. By keeping the same duty cycle and decreasing the period, the oscillations can be made smaller and smaller, and the trajectories of the switched model (1) will converge to that of the averaged model, as depicted in Fig. 2. A second observation follows: for not sufficiently high frequency, the fixed point of the averaged model can be far from the actual limit cycle of the power converter. Therefore, in general, the switched model (1) can describe the evolution of voltage and current in a real converter better than its corresponding averaged model. From here the focus of this work on controlling such switched models.

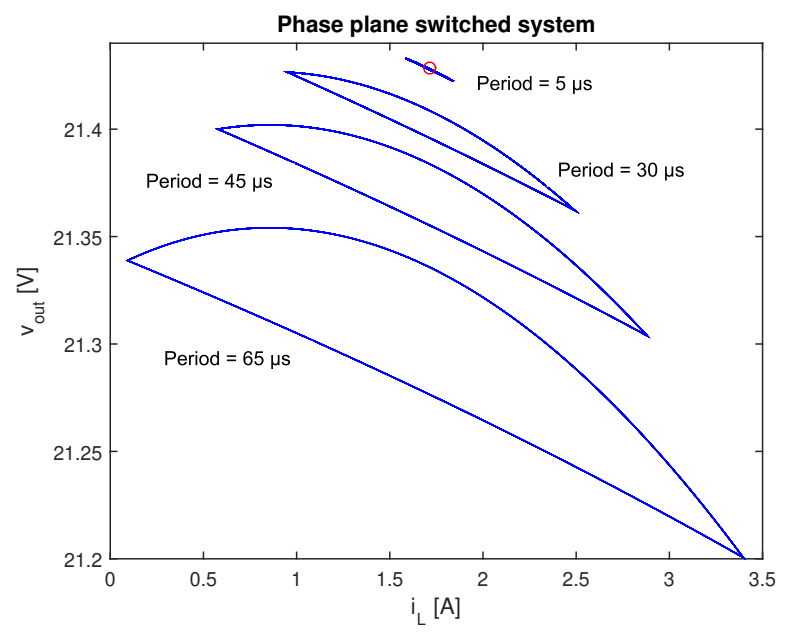

Figure 2: Limit cycle for $D=0.5$ and different periods (the equilibrium of the averaged model is indicated with a circle)

\subsection{Switched dynamics, parametric uncertainty and related work}

The last years have witnessed the increasing interest of the scientific community in the study of switched systems $[6,7,8,9,10,11]$, with applications to power converters, see $[12,13,14,15]$ and reference therein. Most approaches for stability and stabilization of switched systems are based on deriving common or multiple Lyapunov functions, resulting from the solution of linear matrix inequalities (LMIs). When considering power converters however, some peculiar characteristics of such devices should be considered: in [12] a set of attainable equilibrium points that can be reached from any initial condition of the power converter is calculated, and a switched control technique is developed to reach those equilibria. However, in [12] asymptotic stability is considered, whereas Figs. 1(c) and 1(d) reveal that, due to finite switching frequency, no desired state can be reached asymptotically. In fact, as recognized in [13], asymptotic stability requires null intervals between switching times: to ensure a minimum time until switching, [13] uses a delay or a dead zone. The technique in [14] can also ensure a minimum time between switching, since a discrete-time approach is used to characterize the power converter limit cycle: the limit cycle is viewed as an attractor for which stability results are established. The PWM approach in [15] results in a practically stable strategy, where a sliding-mode-like strategy is used till a uniform lower bound on the dwell time is attained.

While capturing the essence of the power converter switched behavior, the aforementioned works neglect a crucial point: a relevant aspect of an effective power converter control law is robustness against 
parameter uncertainty. Even two 'identical' power converters will be subject to process variance intrinsic to device fabrication. Therefore, parametric uncertainty should be taken into account by the control design. Parametric uncertainty can be tackled either via a robust $[16,17,18,19,20,21]$ or via an adaptive $[22,23,24,25,26,27]$ control approach. Using an averaged model, in [16] a robust linear quadratic regulation (LQR) problem is formulated via LMIs, while in $[17,18,19]$ the minimization of the $\mathscr{H}_{2}$ or $\mathscr{H}_{\infty}$ norms is carried out so as to assure a guaranteed region of stability. Using a switched model, constrained stabilization of buck converters based on controlled contractive sets have been considered in [20], while model predictive control is considered in [21]. In most robust control approaches the uncertainty set is represented by a polytope inside which admissible values of the uncertain parameters are constrained. However, as the uncertainty increases (in terms of number of uncertain parameters or size of the uncertainty), lower performance level can be assured. For this reason, in robust control techniques, the uncertainty is limited to a couple of parameters, typically the resistance load and the nominal duty cycle. Adaptive control strategies may deal with larger parametric uncertainties than robust control. In [22] a model reference adaptive controller for the averaged model of a full-bridge buck inverter has been proposed based on minimal controller synthesis. Bilinear dynamics of averaged power converter models have been handled, e.g. via fuzzy neural network control [23] or in the energy-shaping Hamiltonian framework [24]. Other adaptive control techniques include adaptive backstepping [25], dead-beat self-tuning [26], or a combination of adaptive and stabilizing compensators [27]. The work in [28] sits at the boundary between adaptive and robust control, where a parameter-independent (and thus adaptive by definition) strategy is used for transient control, while a robust Proportional-Integral-Derivative (PID) controller is used after the transient: robustness is implicitly assumed as the PID is in charge of stabilizing the state around several possible working points.

While adaptive control has been considered for uncertain but ideal components, an additional source of uncertainty in power converters is given by parasitic effects (e.g. non-ideal switch and diode). The influence of parasitic elements is relevant in terms of final equilibrium value (as shown in Fig. 1(c)). The problem with parasitic effects is that they are in general not known a priori: therefore, model construction via online estimation combined with adaptive control turns out to be a reasonable approach. However, all the aforementioned adaptive approaches use averaging models, and adaptive control using a switched model of the power converter is to the best of the authors' knowledge still an open problem, which motivates this study. In this study we propose an adaptive switching strategy for power converters: the main contribution are: (a) we overcome classical dwell-time ideas in favour of a (reverse) mode-dependent dwell-time strategy that can explicitly define a duty cycle with limited switching frequency; (b) while available sliding-mode or switched limit cycles approaches assume ideal circuit components, in this work we take into account the non-ideal limit cycle behavior of the switched system; (c) while state-of-the-art adaptive approaches handle uncertainties in the averaged model, parametric uncertainty of the switched model (1) is considered here.

The approach pursued in this work can be summarized as follows: at first, we illustrate stability criteria for global uniformly ultimately boundedness (GUUB) around the desired state under reverse modedependent dwell time (Section 2), and we cover the parametric estimation of the switched model (Section 3); after formulating the control problem based on an elementary time unit approach (Section 4), a set of LMIs for GUUB is solved online in an adaptive fashion under the assumption verified in practice of a persistently exciting input voltage source, which guarantees convergence of the estimated parameters to the true system parameters (Section 5). Simulations (Section 6) and conclusions (Section 7) are finally presented.

\section{Notation and preliminary definitions}

The purpose of a control strategy in power converters is to decide the time-dependent PWM strategy $\sigma(\cdot)$ switching between the two modes in (1). Therefore, having in mind the mechanism through which 
convergence to a limit cycle is achieved in power converters, it is important to describe the multiple Lyapunov function tools with which such convergence can be characterized. Most stability results via multiple Lyapunov functions consider regulation to zero of switched systems with stable subsystems, and consider that switching can be a factor destroying stability: therefore, one should stay enough time on a subsystem before switching to the next one, e.g. according to some dwell-time constraint [7].

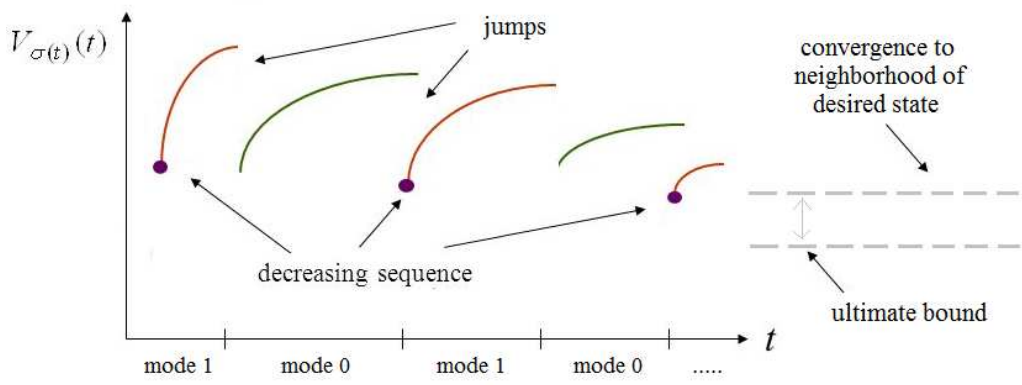

Figure 3: Multiple Lyapunov functions for power converters.

However, the peculiarity of power converters is that the desired state is in general not a fixed point of the single subsystems (cf. Figs. 1(a) and 1(b)): as a consequence, the flow of the single subsystems around the desired state is not 'stable'. Despite that, we know that an appropriate switching can stabilize the system (cf. Fig. 1(c)): this implies that switching can be an enabling factor for stability. This is illustrated by Fig. 3: the figure reveals that despite the fact that a Lyapunov function might increase in between switches, the jump effect can compensate for the possible increase, so as to obtain a sequence converging to a neighborhood of the desired state. Fig. 3 illustrates the a reverse dwell time concept (first introduced in [29]), formalizing the fact that switch cannot be 'too slow'. However, classical reverse dwell-time signals are unable to specify a duty cycle [7]: therefore, it is functional to introduce the following extended dwell-time definition:

Definition 1. [Reverse mode-dependent dwell time [29, 30]] We say that the switching signal $\sigma(\cdot)$ has a reverse mode-dependent dwell time $T_{p}, p \in\{1,0\}$, if after the $p$-th subsystem is activated there is a time no larger than $T_{p}$ before another mode is activated.

Let us consider a fixed switching frequency $1 / T$ for the power converter: since the switching signal can be completely determined by the designer, we assume that subsystems are activated exactly at the maximum allowed reverse mode-dependent dwell time $T_{p}$. Then, it is straightforward to define the duty cycle in terms of mode-dependent dwell time as follows. Assume the power converter starts in the on-state at time $0(\sigma(0)=1)$, and there is a time $T_{1}$ before activating the off-state $\left(\sigma\left(T_{1}\right)=0\right)$, followed by a time $T_{0}$ before activating the on-state again $\left(\sigma\left(T_{1}+T_{0}\right)=1\right)$, and so on periodically: then the period is defined as $T=T_{1}+T_{0}$ and the duty cycle is defined as $T_{1} / T$. From now on, we will fix the period $T$ of the PWM strategy, so that determining $T_{1}$ will automatically determine $T_{0}$ as well.

The objective of a power converter is to track a certain output voltage: as presented in the introduction, the peculiarity of power converters is that the output voltage is approached with some $T$-dependent bounded error by switching between the on and off mode. Thus, it makes sense to define the following concept of global uniformly ultimately boundedness:

Definition 2. [Global uniformly ultimately boundedness [31]] The solutions of a system are global uniformly ultimately bounded (GUUB) with respect to a point $x_{d e s}$ with ultimate bound $\varepsilon$ if there exist positive 
constants $\varepsilon$ and $\delta$, independent of $t_{0} \geq 0$, and for every $\bar{\varepsilon} \in(0, \delta)$, there is $\bar{t}=\bar{t}(\bar{\varepsilon}, \varepsilon)$, also independent of $t_{0}$, such that

$$
\left\|x\left(t_{0}\right)-x_{\text {des }}\right\| \leq \bar{\varepsilon} \Rightarrow\left\|x\left(t, x_{0}\right)-x_{\text {des }}\right\| \leq \varepsilon, \quad \forall t \geq t_{0}+\bar{t}
$$

and, furthermore, (2) holds for arbitrarily large $\bar{\varepsilon}$.

In the power converter application, the smaller the switching period $T$, the smaller the minimum achievable $\varepsilon$ in (2). With some abuse of the definition, whenever (2) holds, we will say that $x_{\text {des }}$ is GUUB. Global uniformly ultimately boundedness under reverse mode-dependent dwell time can be characterized using Lyapunov analysis as stated in the following lemma:

Lemma 1. Consider the switched system (1) and a switching sequence $\left\{t_{0}, t_{1}, \ldots, t_{k}, t_{k+1}, \ldots\right\}$ generated by $\sigma(\cdot)$ under a PWM strategy. If there exist a pair of continuously differentiable, positive definite, radially unbounded Lyapunov functions $W_{p}: \mathbb{R}^{n} \rightarrow \mathbb{R}_{+}, p \in\{1,0\}$, functions $\underline{a}, \bar{a} \in \mathscr{K}_{\infty}$, and pairs of positive constants $\lambda_{p}, \mu_{p}$, and $c_{p}, p \in\{1,0\}$ such that

$$
\begin{aligned}
& \underline{a}\left(\left|x-x_{\text {des }}\right|\right) \leq W_{p}\left(t, x-x_{\text {des }}\right) \leq \bar{a}\left(\left|x-x_{\text {des }}\right|\right), \quad p \in\{1,0\} \\
& \dot{W}_{p}\left(t, x-x_{\text {des }}\right) \leq \lambda_{p} W_{p}\left(t, x-x_{\text {des }}\right)+c_{p}, \quad p \in\{1,0\} \\
& W_{q}\left(t_{k}^{+}, x-x_{\text {des }}\right) \leq \mu_{p} W_{p}\left(t_{k}^{-}, x-x_{\text {des }}\right), \quad p \neq q \in\{1,0\} \\
& \ln \mu_{1}+\ln \mu_{0}+\lambda_{1}\left(t_{k+1}-t_{k}\right)+\lambda_{0}\left(t_{k+2}-t_{k+1}\right)<0, \quad k \in \mathbb{N}_{+}
\end{aligned}
$$

where $t_{k}^{-}$and $t_{k}^{+}$indicate the time instant right before and right after the switching time $t_{k}$ then, the state $x_{\text {des }}$ of the switched system (1) is GUUB.

Proof. See Appendix B for the complete proof. A sketch of the proof is: consider the Lyapunov function $W_{\sigma(t)}\left(t, x(t)-x_{d e s}\right)$. The positive $\lambda_{p}$ in the second inequality of (3) allows the Lyapunov function to increase in between switching instants, while the third inequality of (3) allows the Lyapunov function to decrease at switching instants (if $0<\mu_{p}<1$ ) or to bound its increase (if $\mu_{p}$ is slightly larger than 1). The fourth inequality of (3) allows us to select $\lambda_{p}$ and $\mu_{p}$ is such a way that increase/decrease mechanism results, overall, in an exponentially decreasing sequence till a bound $\varepsilon$ around $x_{d e s}$ depending on $c_{p}$ is reached. The level sets of the Lyapunov function can be used to derive the ultimate bound $\varepsilon$ guaranteeing $\left\|x-x_{d e s}\right\| \leq \varepsilon$ (cf. the details in Appendix B for an estimate of $\varepsilon$ ).

Remark 1. Lemma 1 holds for switched models of power converters in the form (1), in view of the existence of limit cycles [1,2]. In particular, in contrast with linear designs based on average nonlinear (i.e. bilinear) models of power converters which lead to a finite region of attraction [16, 17], Lemma 1 results in global stability and attraction results (valid for the entire state space). With respect to the state of the art, e.g. Theorem 1 in [32], which also uses an increase/decrease mechanism as illustrated in Fig. 3, Lemma 1 improves the following points: instead of a reverse dwell time, a reverse mode-dependent dwell time is adopted, so that a duty cycle can be specified; in addition, the last inequality in (3) does not require $\ln \mu_{1}+$ $\lambda_{1}\left(t_{k+1}-t_{k}\right)<0$ and $\ln \mu_{0}+\lambda_{0}\left(t_{k+2}-t_{k+1}\right)<0, k \in \mathbb{N}_{+}$as in in [32]. In other words, the constants $\mu_{p}$, $p \in\{1,0\}$ are not required to be both in between 0 and 1 .

Remark 2. It is clear that according to Lemma 1, it suffices to take $c_{p}$ large enough to prove GUUB: this would lead to a large bound $\varepsilon$ in (2). Therefore, for Lemma 1 to be of practical relevance, one should look for the smallest $c_{p}$ such that (3) holds, so as to obtain a small bound $\varepsilon$ around $x_{d e s}$.

In the following we will explain how to deal with parametric uncertainties, a situation faced very often in real applications when all the circuit parameters (inductances, capacitances, resistances) are uncertain or even completely unknown (note that parasitic resistances are extremely difficult to be known). 


\footnotetext{
${ }^{1}$ Here and in the following, time dependence of the state, input and switching signals might be omitted when obvious.

${ }^{2}$ This implies that the desired state is well-defined so that the duty cycle is not always 0 or 1 , i.e. $v_{\text {out }}$ ref $>v_{s}$ with $v_{\text {out }}$ ref bounded for a boost converter, and $v_{\text {out }}<v_{s}$ with $v_{\text {out }}$ ref far from 0 for a buck converter.
}

In this work we will refer to the boost converter (A.1), (A.2) in Appendix A: however, similar reasoning applies to any converter (e.g. buck and buck-boost) which can be written as a switched system in the form ${ }^{1}$

$$
\dot{x}=A_{\sigma} x+E_{\sigma} v_{s}
$$

where $x=\left[\begin{array}{ll}i_{L} & v_{\text {out }}\end{array}\right]^{\prime}$ and $\sigma$ is the time-dependent PWM switching signal to be determined. Note that the models in (A.1), (A.2) contain 8 uncertain parameters $\left(R_{D 0}, R_{D 1}, R_{S 0}, R_{S 1}, C, L, R_{L}, R_{\text {load }}\right)$. In order to obtain a model which is linear with respect to the parameters, we reparametrize the system by estimating all the nonzero entries of $A_{1}, B_{1}, A_{0}, B_{0}$ in (A.1), (A.2). More precisely, considering that the modes share some parameters, we have 9 parameters to be estimated (cf. (A.4) in Appendix A). The parameter estimation task will be performed with standard techniques from parameter estimation. To this purpose we write (4) as

$$
\dot{x}=A_{m} x+\left(A_{\sigma}-A_{m}\right) x+E_{\sigma} v_{s},
$$

with $A_{m}$ a given Hurwitz matrix. We use the series-parallel parametric model [33, Sect. 4.2.2] to obtain

$$
\dot{\hat{x}}=A_{m} \hat{x}+\left(\hat{A}_{\sigma}-A_{m}\right) x+\hat{E}_{\sigma} v_{s},
$$

where $\hat{x}$ is the state of the parametric model and $\hat{A}_{p}, \hat{E}_{p}$ are the matrices to be estimated, for each mode $p \in\{1,0\}$. Define $\Theta_{\sigma}^{*}=\operatorname{vec}\left[A_{\sigma} E_{\sigma}\right]^{\prime}, \hat{\Theta}_{\sigma}=\operatorname{vec}\left[\hat{A}_{\sigma} \hat{B}_{\sigma}\right]^{\prime}, \phi=\left[x^{\prime} v_{s} 0 ; 0 x^{\prime} v_{s}\right]$ and adopt a parameter estimator based on integral cost and gradient update [33, Sect. 4.3.5]

$$
\dot{\hat{\Theta}}_{\sigma}=\mathrm{P}\left(-\gamma \bar{R}_{\sigma} \hat{\Theta}_{\sigma}-\gamma \bar{Q}_{\sigma}\right), \quad \hat{\Theta}_{\sigma}(0)=\Theta_{0}
$$

$$
\dot{\bar{R}}_{\sigma}=-\beta \bar{R}_{\sigma}+\phi_{f} \phi_{f}^{\prime}, \quad \bar{R}_{\sigma}(0)=0
$$

$$
\dot{\bar{Q}}_{\sigma}=-\beta \bar{Q}_{\sigma}-\phi_{f}(x-g), \quad \bar{Q}_{\sigma}(0)=0
$$

$$
\dot{\phi}_{f}=A_{m} \phi_{f}+\phi, \quad \phi_{f}(0)=0
$$

$$
\dot{g}=A_{m} g-A_{m} x, \quad g(0)=x(0),
$$

where $\beta$ and $\gamma$ are positive constants and $\mathrm{P}$ denotes a projection operator which has to be designed to keep the estimates inside a convex set: such convex set is known a priori if some bounds for the parameters $\left(R_{D 0}\right.$, $R_{D 1}, R_{S 0}, R_{S 1}, C, L, R_{L}, R_{\text {load }}$ ) are known. The following convergence result can be stated.

Theorem 1. Consider the switched model (4) of a power converter, driven by a well-posed PWM signal ${ }^{2}$. Provided that the input voltage $v_{s}$ is bounded, the estimation law (7) satisfies the following properties:

i) $\lim _{t \rightarrow \infty} x(t)-\hat{x}(t)=0$

ii) $\lim _{t \rightarrow \infty}\left|\dot{\hat{\Theta}}_{\sigma}(t)\right|=0$

iii) if $\phi(\cdot)$ is persistently exciting and $\sigma(\cdot)$ has nonzero activation time in both modes, then $\hat{\Theta}_{\sigma(t)}(t) \rightarrow$ $\Theta_{\sigma(t)}^{*}$ exponentially on the respective activation time, and the rate of convergence increases with $\gamma$.

Proof. This result can be derived by straightforwardly extending the results in [33, Thm. 4.3.3] on the respective activation times of mode 1 and mode 0 . Note that, under a well-posed PWM strategy, the switching signal $\sigma(\cdot)$ will 'visit both modes', i.e. both modes will have nonzero activation time. 


\section{Problem formulation}

In this section we formulate the power converter control problem as a stabilization problem for switched systems. First, we define the reference to be tracked, i.e. the desired output voltage and inductor current:

$$
x_{\text {des }}=\left[\begin{array}{c}
i_{L_{d e s}} \\
v_{\text {out }} t_{\text {des }}
\end{array}\right] \text {. }
$$

A common approach for the definition of the desired inductor current $i_{L_{d e s}}$, which will be pursued here, is to calculate it assuming that the power delivered by the source is consumed by the load resistance only (i.e. lossless case) [34]. Note that, since $i_{L_{d e s}}$ might depend on some unknown circuit parameters, an estimate $\hat{x}_{d e s}$ must be used. We are now ready to give the problem formulation:

Problem 1. [Adaptive PWM design] Given the switched affine model (1) of a power converter with uncertain matrix entries, design a PWM strategy that combines the online estimator (7) with the reverse modedependent switching of Lemma 1, and such that the solutions of the resulting system are GUUB with respect to $x_{\text {des }}$, for any initial state and regardless of the operating point and uncertainty. Furthermore, for a given switching period $T$, derive (and minimize) the ultimate bound as a function of design parameters.

\subsection{Elementary time unit approach}

To formulate a sufficient condition for GUUB of the switched model of the power converter, we will constructively find a multiple Lyapunov function and a reverse mode-dependent dwell time switching sequence such that Lemma 1 is verified. We make use of stability criteria which are convex with respect to the power converter matrices (convexity is required to prove stability of the adaptive closed-loop): this is possible by using a mode-dependent version of the so-called elementary time unit approach [32, 35]. The elementary time unit approach uses multiple positive definite matrices for each subsystem to construct a time-varying Lyapunov function. For example, with positive definite matrices $P_{1}^{[l]}, P_{0}^{[l]}, l=1, \cdots, L$, the mode-dependent dwell time is partitioned such that $T_{1}=\sum_{l=0}^{L-1} \delta_{T_{1}}^{[l]}, T_{0}=\sum_{l=0}^{L-1} \delta_{T_{0}}^{[l]}$, and the Lyapunov function $V_{p}(x(t))=\left(x(t)-x_{d e s}\right)^{\prime} P_{p}(t)\left(x(t)-x_{d e s}\right)$ for subsystem $p \in\{1,0\}$ is constructed as

$$
P_{p}(t)= \begin{cases}P_{p}^{[l]}+\left(P_{p}^{[l+1]}-P_{p}^{[l}\right) \frac{t-t_{k}-\sum_{r=0}^{l-1} \delta_{T_{p}}^{[r]}}{\delta_{p}^{[l]}} & t_{k}+\sum_{r=0}^{l-1} \delta_{p}^{[r]} \leq t<t_{k}+\sum_{r=0}^{l} \delta_{p}^{[r]} \\ P_{p}^{[L]} & t=t_{k}+T_{p}=t_{k+1}\end{cases}
$$

where $t_{k}$ is the time instant when subsystem $p$ becomes active and $t_{k+1}$ is the time instant when the other subsystem becomes active: (8) means that the matrix $P_{p}(t)$ results from the linear interpolation of $P_{p}^{[l]}$, $l=1, \cdots, L$ during the mode-dependent dwell time. To simplify the notation, let us define $F_{1}=A_{1} x_{d e s}+E_{1} v_{s}$ and $F_{0}=A_{0} x_{d e s}+E_{0} v_{s}$. The following global uniformly ultimately boundedness result is shown:

Theorem 2. Assume that for some $0<T_{1}<T$ (and consequently $T_{0}=T-T_{1}>0$ ), there exist positive 
definite matrices $P_{1}^{[l]}, P_{0}^{[l]}, l=1, \cdots, L$, and pairs of positive constants $\lambda_{1}, \lambda_{0}, \mu_{1}, \mu_{0}$, and $c_{1}, c_{0}$ such that

$$
\begin{aligned}
& {\left[\begin{array}{cc}
\frac{P_{1}^{[l+1]}-P_{1}^{[l]}}{\delta_{T_{1}}^{[l]}}+A_{1}^{\prime} P_{1}^{[l]}+P_{1}^{[l]} A_{1}-\lambda_{1} P_{1}^{[l]} & P_{1}^{[l]} F_{1} \\
F_{1}^{\prime} P_{1}^{[l]} & -c_{1}
\end{array}\right]<0} \\
& {\left[\begin{array}{cc}
\frac{P_{1}^{[l+1]}-P_{1}^{[l]}}{\delta_{T_{1}}^{[l]}}+A_{1}^{\prime} P_{1}^{[l+1]}+P_{1}^{[l+1]} A_{1}-\lambda_{1} P_{1}^{[l+1]} & P_{1}^{[l+1]} F_{1} \\
F_{1}^{\prime} P_{1}^{[l+1]} & -c_{1}
\end{array}\right]<0, \quad l=1, \cdots, L-1} \\
& {\left[\begin{array}{cc}
A_{1}^{\prime} P_{1}^{[L]}+P_{1}^{[L]} A_{1}-\lambda_{1} P_{1}^{[L]} & P_{1}^{[L]} F_{1} \\
F_{1}^{\prime} P_{1}^{[L]} & -c_{1}
\end{array}\right]<0} \\
& {\left[\begin{array}{cc}
\frac{P_{0}^{[l+1]}-P_{0}^{[l]}}{\delta_{T_{0}}^{l l]}}+A_{0}^{\prime} P_{0}^{[l]}+P_{0}^{[l]} A_{0}-\lambda_{0} P_{0}^{[l]} & P_{0}^{[l]} F_{0} \\
F_{0}^{\prime} P_{0}^{[l]} & -c_{0}
\end{array}\right]<0} \\
& {\left[\begin{array}{cc}
\frac{P_{0}^{[l+1]}-P_{0}^{[l]}}{\delta_{T_{0}}^{l]}}+A_{0}^{\prime} P_{0}^{[l+1]}+P_{0}^{[l+1]} A_{0}-\lambda_{0} P_{0}^{[l+1]} & P_{0}^{[l+1]} F_{0} \\
F_{0}^{\prime} P_{0}^{[l+1]} & -c_{0}
\end{array}\right]<0, \quad l=1, \cdots, L-1} \\
& {\left[\begin{array}{cc}
A_{0}^{\prime} P_{0}^{[L]}+P_{0}^{[L]} A_{0}-\lambda_{0} P_{0}^{[L]} & P_{0}^{[L]} F_{0} \\
F_{0}^{\prime} P_{0}^{[L]} & -c_{0}
\end{array}\right]<0} \\
& \mu_{1} P_{1}^{[L]}-P_{0}^{[1]}>0 \\
& \mu_{0} P_{0}^{[L]}-P_{1}^{[1]}>0 \\
& \ln \mu_{1}+\ln \mu_{0}+\lambda_{1} T_{1}+\lambda_{0} T_{0}<0,
\end{aligned}
$$

then a switching law with reverse mode-dependent dwell time $T_{1}, T_{0}$ makes the state $x_{\text {des }} G U U B$.

Proof. The proof amounts to showing that Theorem 2 satisfies all the conditions of Lemma 1. The Lyapunov function is of the form $V\left(t, x(t)-x_{\text {des }}\right)=\left(x(t)-x_{d e s}\right)^{\prime} P_{\sigma(t)}(t)\left(x(t)-x_{d e s}\right)$, therefore the conditions $\underline{a}\left(\left|x-x_{\text {des }}\right|\right) \leq V_{p}\left(t, x-x_{\text {des }}\right) \leq \bar{a}\left(\left|x-x_{\text {des }}\right|\right)$ are satisfied for some quadratic functions $\underline{a}, \bar{a}$ depending on $\bar{\lambda}=$ $\max _{p} \lambda_{\max }\left(P_{p}\right)$ and $\underline{\lambda}=\min _{p} \lambda_{\min }\left(P_{p}\right)$ (being $\lambda_{\max }(\cdot)$ and $\lambda_{\min }(\cdot)$ the maximum and minimum eigenvalues, respectively). In addition, the first six LMIs in (9) are equivalent to $\dot{V}_{p}\left(t, x-x_{d e s}\right) \leq \lambda_{p} V_{p}\left(t, x-x_{d e s}\right)+c_{p}$. Then, the seventh and eighth LMIs in (9) are equivalent to $V_{q}\left(t_{k}^{+}, x-x_{d e s}\right) \leq \mu_{p} V_{p}\left(t_{k}^{-}, x-x_{d e s}\right)$. Finally, the last inequality in (9) is the same as the last in Lemma 1. Therefore, the Lyapunov function $V\left(t, x(t)-x_{d e s}\right)$ satisfies the conditions of Lemma 1, from which GUUB of the state $x_{d e s}$ of the switched system (1) is derived. Finally, an estimate of the ultimate bound is $\varepsilon=\bar{c} \sqrt{\bar{\lambda}} / \underline{\lambda}$, where $\bar{c}=c_{1}+c_{0}$. Therefore, similarly to Lemma 1, in order to obtain a small $\varepsilon$, Theorem 2 should be solved for the minimum constants $c_{1}$ and $c_{0}$, which are linear variables to be optimized in (9).

Remark 3. The elementary time unit approach of Theorem 2 leads to a problem which is linear (i.e. convex) with respect to the power converter matrices: on the other hand, the computational complexity of the LMIs in Theorem 2 increases for increasing L. Similarly to standard multiple Lyapunov function stability criteria, in order for (9) to be LMIs, the constants $\lambda_{p}, \mu_{p}, p \in\{1,0\}$ should be fixed by the designer or found by some line search strategy. 


\section{Adaptive control strategy}

The global uniformly ultimately boundedness theorem developed in the previous section assumes that the system matrices are known. In this section we will shown how it is possible to develop an iterative algorithm where the system matrices are estimated from data and a set of linear matrix inequalities solved online. In words, the proposed control scheme iterates the following phases: a) Stability step (GUUB based on the current estimated system); b) Lyapunov-based switching step (based on the reverse mode-dependent dwell time); c) Estimation step (online). The following paragraphs explain each one of these phases.

a) Stability step: Let us assume that at time $t$ the estimates $\hat{\Theta}_{1}$ and $\hat{\Theta}_{0}$ are given, which contain the estimated matrices $\hat{A}_{1}, \hat{E}_{1}, \hat{A}_{0}$ and $\hat{E}_{0}$. Given the objective of global uniformly ultimately boundedness around the desired state desired state $x_{\text {des }}$, the choice of the performance objectives passes through the definition of the duty cycle of the power converter $T_{1} / T$ with $0<T_{1}<T$. After fixing the period $T$, one would like the reverse mode-dependent switching strategy that minimizes the bound $\varepsilon$ on the error with respect to the desired state (where $\varepsilon$ is as defined in the proof of Theorem 2): this is accomplished by solving the following set of LMIs

$$
\begin{aligned}
& \min c_{1}+c_{0} \\
& \text { s.t. } \\
& {\left[\begin{array}{cc}
\frac{P_{1}^{[l+1]}-P_{1}^{[l]}}{\delta_{T_{1}}^{l]}}+\hat{A}_{1}^{\prime} P_{1}^{[l]}+P_{1}^{[l]} \hat{A}_{1}-\lambda_{1} P_{1}^{[l]} & P_{1}^{[l]} \hat{F}_{1} \\
\hat{F}_{1}^{\prime} P_{1}^{[l]} & -c_{1}
\end{array}\right]<0} \\
& {\left[\begin{array}{cc}
\frac{P_{1}^{[l+1]}-P_{1}^{[l]}}{\delta_{T_{1}}^{l l]}}+\hat{A}_{1}^{\prime} P_{1}^{[l+1]}+P_{1}^{[l+1]} \hat{A}_{1}-\lambda_{1} P_{1}^{[l+1]} & P_{1}^{[l+1]} \hat{F}_{1} \\
\hat{F}_{1}^{\prime} P_{1}^{[l+1]} & -c_{1}
\end{array}\right]<0, \quad l=1, \cdots, L-1} \\
& {\left[\begin{array}{cc}
\hat{A}_{1}^{\prime} P_{1}^{[L]}+P_{1}^{[L]} \hat{A}_{1}-\lambda_{1} P_{1}^{[L]} & P_{1}^{[L]} \hat{F}_{1} \\
\hat{F}_{1}^{\prime} P_{1}^{[L]} & -c_{1}
\end{array}\right]<0} \\
& {\left[\begin{array}{cc}
\frac{P_{0}^{[l+1]}-P_{0}^{[l]}}{\delta_{T_{0}}^{l l}}+\hat{A}_{0}^{\prime} P_{0}^{[l]}+P_{0}^{[l]} \hat{A}_{0}-\lambda_{0} P_{0}^{[l]} & P_{0}^{[l]} \hat{F}_{0} \\
\hat{F}_{0}^{\prime} P_{0}^{[l]} & -c_{0}
\end{array}\right]<0} \\
& {\left[\begin{array}{cc}
\frac{P_{0}^{[l+1]}-P_{0}^{[l]}}{\delta_{T_{0}}^{[l]}}+\hat{A}_{0}^{\prime} P_{0}^{[l+1]}+P_{0}^{[l+1]} \hat{A}_{0}-\lambda_{0} P_{0}^{[l+1]} & P_{0}^{[l+1]} \hat{F}_{0} \\
\hat{F}_{0}^{\prime} P_{0}^{[l+1]} & -c_{0}
\end{array}\right]<0, \quad l=1, \cdots, L-1} \\
& {\left[\begin{array}{cc}
\hat{A}_{0}^{\prime} P_{0}^{[L]}+P_{0}^{[L]} \hat{A}_{0}-\lambda_{0} P_{0}^{[L]} & P_{0}^{[L]} \hat{F}_{0} \\
\hat{F}_{0}^{\prime} P_{0}^{[L]} & -c_{0}
\end{array}\right]<0} \\
& \mu_{1} P_{1}^{[L]}-P_{0}^{[1]}>0 \\
& \mu_{0} P_{0}^{[L]}-P_{1}^{[1]}>0 \\
& \ln \mu_{1}+\ln \mu_{0}+\lambda_{1} T_{1}+\lambda_{0} T_{0}<0
\end{aligned}
$$

where $\hat{F}_{1}=\hat{A}_{1} \hat{x}_{d e s}+\hat{E}_{1} v_{s}$ and $\hat{F}_{0}=\hat{A}_{0} \hat{x}_{d e s}+\hat{E}_{0} v_{s}$. Upon fixing $T$, a bisection algorithm is required to find $T_{1}$ and thus the duty cycle $T_{1} / T$. The criterion driving the bisection algorithm is defined by checking whether a smaller $\varepsilon$ than the previous iteration can be attained. The LMIs (10) guarantee that for the estimated system a switching law with mode-dependent dwell time $T_{1}, T_{0}$ makes the desired state $\hat{x}_{\text {des }}$ GUUB. 
b) Lyapunov-based switching step: The switching step consists of two parts: first, the duty cycle $T_{1} /\left(T_{1}+T_{0}\right)$ defined by the mode-dependent dwell time is implemented; second, a small dither $\tilde{v}_{s}$ is added to the input voltage is such a way that persistency of excitation is guaranteed:

$$
v_{s}^{o}(t)=v_{s}+\arg \min _{v_{s} \in \Xi}\left\{\frac{\partial V_{\sigma(t)}}{\partial t}+\frac{\partial V_{\sigma(t)}}{\partial x}\left[\hat{A}_{\sigma(t)} x+\hat{E}_{\sigma(t)}\left(v_{s}+\tilde{v}_{s}\right)\right]-\lambda_{\sigma(t)} V_{\sigma(t)}-c_{\sigma(t)}\right\}
$$

where $V_{\sigma(t)}(t, x(t))=\left(x-x_{d e s}\right)^{\prime} P_{\sigma(t)}(t)\left(x-x_{d e s}\right)$ comes from the solution of (10), and

$$
\Xi=\{\tilde{v} \mid \tilde{v} \in[-2 \alpha,-\alpha] \cup[\alpha, 2 \alpha]\}
$$

being $\alpha$ a user-defined constant that determines the size of the dither. Note that the term inside parentheses in (11) evaluates the Lyapunov-based stability of the system in between switches.

Remark 4. Provided that the estimates $\hat{\Theta}_{1}$ and $\hat{\Theta}_{0}$ are updated online at the same switching frequency of the converter, the solution to the LMIs (10) can be done at lower frequency or in an aperiodic way: when the estimates do not change, or when the trajectory evaluated via (11) is within desired bounds, one can keep the same duty cycle without solving a new set of LMIs.

c) Estimation step: The system is estimated online using (7): the following remark follows.

Remark 5. The dither in (11), (12) guarantees that $\phi$ is persistently exciting: furthermore, since $0<$ $T_{1}<T$, both modes of the power converter are visited with persistency of excitation, and the exponential convergence result in bullet iii) of Theorem 1 holds. Realizing the dither would require a small extra voltage source in series with the inductance. However, in power conversion practice, $v_{s}$ is never perfectly constant and is usually exhibits an uncontrollable ripple which provides already enough persistence of excitation: in such a case, the addition of the dither might be unnecessary.

\subsection{Main result}

Algorithm 1 presents the online procedure for power converter control: the corresponding stability result is given.

Theorem 3. Consider the switched system (1) controlled by Algorithm 1. Then, there exist finite positive constants $\beta_{1}, \beta_{2}, \underline{\gamma}$ and a positive number $\bar{t}=\mathscr{O}\left(\frac{1}{\gamma}\right)$ such that the following holds: if $\alpha$ in (12) satisfies

$$
\begin{cases}0<\alpha \leq \beta_{2} & \text { if } t \leq \bar{t} \text { or }\left|\frac{d V(t)}{d x} \hat{E}_{\sigma(t)}\right| \leq \eta \\ \alpha \geq \beta_{1} & \text { otherwise }\end{cases}
$$

where $V(t)$ is the multiple Lyapunov function available at time $t$ from the solution of (10), $\eta$ is a positive design constant and the adaptive gain $\gamma$ of the estimator satisfies $\gamma \geq \underline{\gamma}$. Then, the proposed adaptive control scheme guarantees bounded closed-loop solutions and, moreover,

$$
\limsup _{t \rightarrow \infty}\left\|x(t)-x_{\text {des }}\right\| \leq \varepsilon_{3},
$$

for a tunable bound $\varepsilon_{3}$, which is affected by the choice of $\beta_{1}, \beta_{2}$ and $\eta$.

Proof. See Appendix C. 


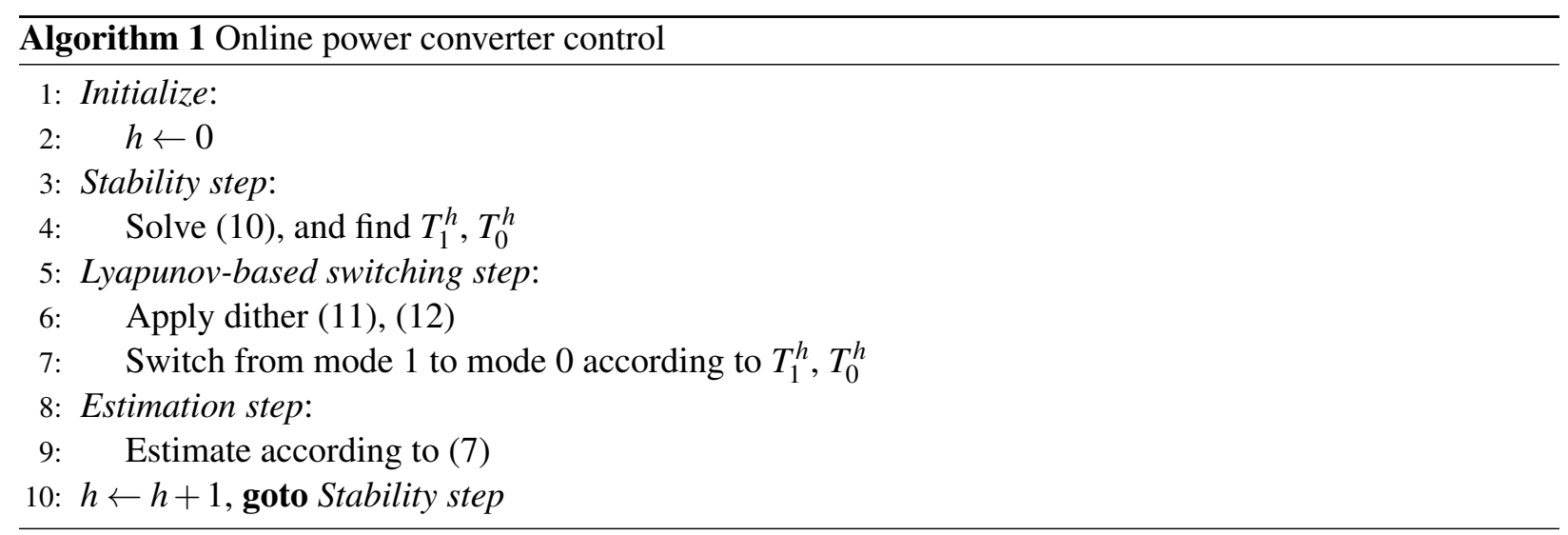

Remark 6. The following comments apply to the design parameters. The time instant $\bar{t}$ is the time required for the estimates to converge close to their actual values (under persistency of excitation). This time can be made smaller and smaller by increasing $\gamma$. The parameter $\eta$ gives an estimate of the region where the Lyapunov sequence decreases exponentially toward the limit cycle: far from the desired state high excitation does not compromise stability, i.e. $\alpha \geq \beta_{1}$; close to the desired state convergence is not exponential anymore, and the excitation should be small enough, i.e. $\alpha \leq \beta_{2}$. In the presence of an uncontrollable ripple in $v_{s}$, Theorem 3 has a practical meaning: there is no benefit in selecting the switching frequency to be too high (equivalently, $T$ too small), since the ultimate bound will be determined also by the ripple in $v_{s}$.

Remark 7. Due to the estimation scheme of Theorem 1, Theorem 3 is valid for the entire parametric uncertainty set. This is contrast with linear control designs for nonlinear (i.e. bilinear) average models, which will in general can be proven to be stable for a finite parametric uncertainty set. Obviously, in Theorem 3 , the learning transient will be influenced by the initial condition for the parameter estimates.

\section{Numerical example}

A boost converter is used for validation, with $v_{s}=12 \mathrm{~V}$, fixed switching period $T=5 \cdot 10^{-6} \mathrm{~s}$, and with circuit parameters taken in line with [16]:

$$
\begin{array}{rlr}
L=100 \cdot 10^{-6} \mathrm{H} & R_{L}=0.5 \Omega & \text { (actual parameters) } \\
R_{D_{1}}=0.5 \Omega & R_{D_{0}}=1 \cdot 10^{9} \Omega & \\
R_{S_{1}}=0.5 \Omega & R_{S_{0}}=1 \cdot 10^{9} \Omega & \\
C=200 \cdot 10^{-6} \mathrm{~F} & R_{\text {load }}=25 \Omega &
\end{array}
$$

As compared to [16], we have included parasitic effects (non-ideal inductor, switch and diode), whose values have been mutuated from [21]. Since we assume that the parameters in (14) are uncertain, a priori knowledge resides in the fact that the proposed adaptive strategy uses the following initial estimates of the parameters

$$
\begin{array}{rll}
L=75 \cdot 10^{-6} \mathrm{H} & R_{L}=0.25 \Omega & \text { (initial estimates) } \\
R_{D_{1}}=0.25 \Omega & R_{D_{0}}=2 \cdot 10^{9} \Omega & \\
R_{S_{1}}=0.25 \Omega & R_{S_{0}}=2 \cdot 10^{9} \Omega & \\
C=300 \cdot 10^{-6} \mathrm{~F} & R_{\text {load }}=15 \Omega &
\end{array}
$$


which are significantly far from the actual parameters. Being $T=5 \cdot 10^{-6} \mathrm{~s}$, the switching frequency of the power converter is $200 \mathrm{kHz}$. With respect to the proposed adaptive approach, the following design parameters have been chosen: $\beta=1 \cdot 10^{-4}, \gamma=1 \cdot 10^{3}, A_{m}=-100 I_{2}, \bar{t}=0.005, \beta_{1}=0.05, \beta_{2}=0.075$, $\eta=0.02$, and for the LMIs we have selected $\lambda_{1}=\lambda_{0}=0.1$ and $\mu_{1}=\mu_{0}=0.98$. As a means of comparison, the robust LQR design in [16], based on the averaged bilinear model of the boost converter is considered. The design leads to a non-adaptive state-feedback gain $K_{L M I-L Q R}=\left[\begin{array}{llll}-0.86 & -1.39 & 3159.54\end{array}\right]$ (the third extra state comes from extending the averaged model with an integrator of the tracking error to attain zero offset, c.f. the design in [16]). The gain $K_{L M I-L Q R}$ has been designed on the actual parameters (14), which require more a priori knowledge than an adaptive controller. In some simulations, we consider initial conditions based on the common practice of power converter control: boost converters are often precharged before control takes over and regulates the output voltage. Precharge can be realized (among other methods) by bypassing the inductor with a precharge diode: this implies that the output voltage is approximately equal to the input voltage when regulation starts [36]. Therefore, for a desired state $v_{\text {out des }}=24 \mathrm{~V}, i_{L_{d e s}}=1.92 \mathrm{~A}$ and the nominal duty cycle $D=0.5$, the precharging initial conditions can be approximately taken as $v_{\text {out des }}(0)=12 \mathrm{~V}, i_{L_{\text {des }}}(0)=3.84 \mathrm{~A}$.

\subsection{Robustness to converter components parametric uncertainty}
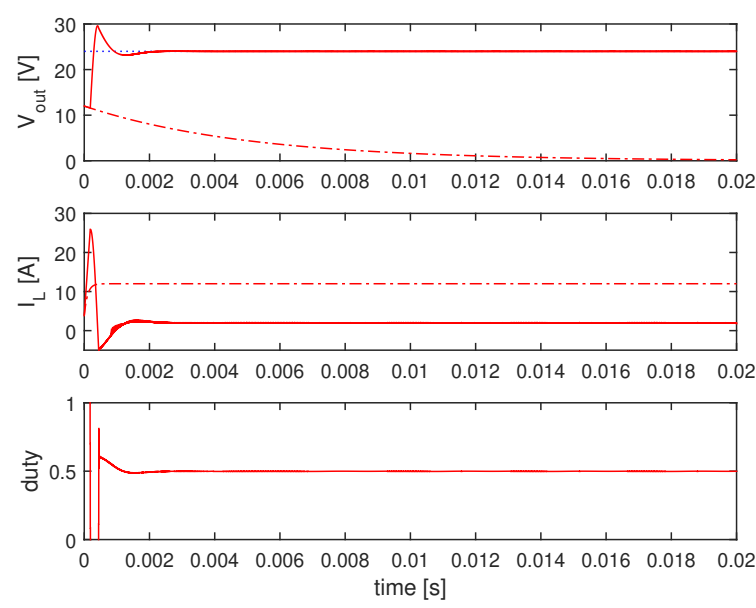

Figure 4: Boost converter with $K_{L M I-L Q R}$ with precharging initial conditions: Output voltage, inductor current and duty cycle for the ideal (non-lossy) converter (solid line) and for the non-ideal (lossy) converter (dash-dotted line). The desired voltage is the dotted line.

Fig. 4 shows that, when the converter components are ideal i.e. without parasitic elements, the controller $K_{L M I-L Q R}$ is capable of starting up the converter from precharging initial conditions (the interested reader can verify that $K_{L M I-L Q R}$ is also capable of starting up the converter from zero initial conditions, indicating that the local region of stability of $K_{L M I-L Q R}$ is quite large). However, despite being designed exactly for the actual parameters (14), the presence of parasitic elements (cf. the model of Appendix A) apparently shrinks the local region of stability: in fact, Fig. 4 indicates that it is impossible for the $K_{L M I-L Q R}$ to reach the desired state when starting from precharging initial conditions and when parasitic elements are introduced ${ }^{3}$.

\footnotetext{
${ }^{3}$ In [16] it is clearly shown how $K_{L M I-L Q R}$ can cope with some components parametric uncertainty and is therefore robust. However, stability of the linear controller is necessarily local, and the region of stability might even shrink if uncertainty is large.
} 

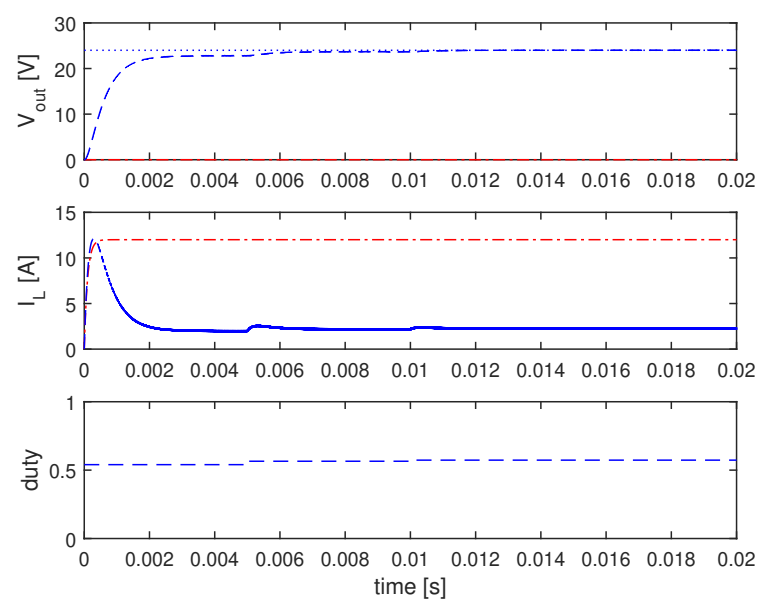

Figure 5: Non-ideal (lossy) boost converter with zero initial conditions: Output voltage, inductor current and duty cycle for $K_{L M I-L Q R}$ (dash-dotted line), and the proposed adaptive strategy (dashed line). The desired voltage is the dotted line.

\footnotetext{
${ }^{4}$ Even though precharging initial conditions are more representative of common practice, zero initial conditions have been used in this simulation to demonstrate global stability properties.
}

In other words, that any linear non-adaptive controller has limited robustness to parametric uncertainty, and has a finite region of stability (which may not include the zero or precharging initial conditions). On the other hand, as expected by the global stability result of Theorem 3, Fig. 5 shows that the proposed adaptive controller, even when starting with the initial estimates (15), handles the parametric uncertainty (including parasitic elements), and is capable of starting up the converter even from zero initial conditions ${ }^{4}$.
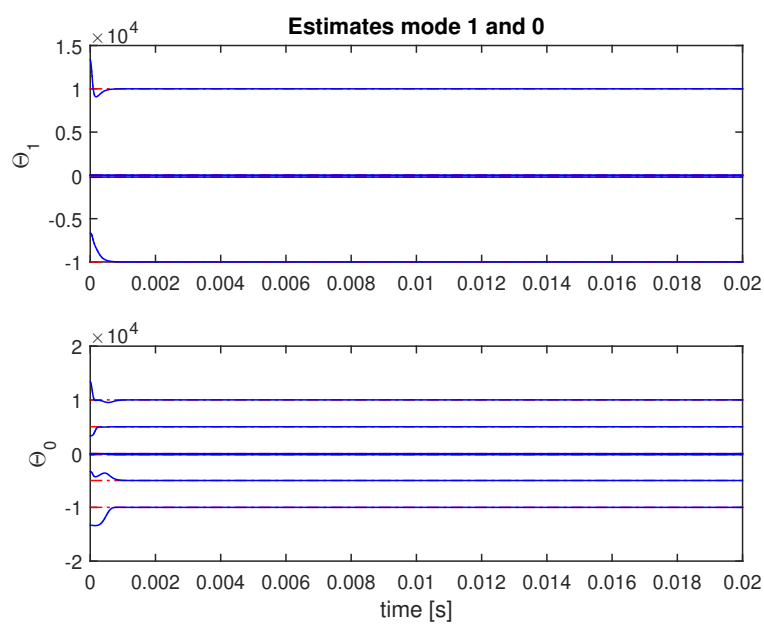

Figure 6: Non-ideal (lossy) boost converter: Online evolution of the parameter estimates for each mode. Estimates (solid line), actual parameters (dash-dotted line).

The key feature of the proposed adaptive approach is the capability of estimating the uncertain parameters: in particular, Fig. 6 shows that the estimator can converge to the actual parameters in less than $1 \mathrm{~ms}$ (cf. Fig. 6). Table 1 elaborates on the number of decision variables and semidefinite inequality constraints 
necessary to solve the LMI problem. The computational complexity of the elementary time unit approach of Theorem 2 would increase for increasing $L$. As a trade-off between conservative results and computational complexity, we apply the elementary time unit approach of Theorem 2 with two positive definite matrices for each subsystem $(L=2)$ : this has been verified to work for the system at hand. It has to be noted that the on-line computational cost of LMIs might be hard for embedded systems: despite some recent advances in SDP solvers for embedded systems [37] it is not yet possible to solve the LMIs periodically at the frequency of hundreds of $\mathrm{kHz}$. Therefore, in the simulations we have assumed the LMIs to be solved at the frequency of $200 \mathrm{~Hz}$ (while the switching frequency of the power converter is kept at $200 \mathrm{kHz}$ ): this leads to a desired settling time (the time at which the converter output has entered and remained within a 5\% error band) of around $10 \mathrm{~ms}$, which is respected by our adaptive approach.

\begin{tabular}{c|c|c}
\hline \hline & No. decision var. & No. constraints \\
\hline \hline Theorem $2, L=2$ & 30 & 14 \\
\hline Theorem $2, L=4$ & 62 & 26 \\
\hline
\end{tabular}

Table 1: Computational cost of LMI solution

\subsection{Robustness to variations of duty cycle}

Fig. 7 shows that, when we start from initial conditions close to the desired state, namely $v_{\text {out }}=23.4 \mathrm{~V}$ and $i_{L}=0 \mathrm{~A}$ (we have verified that selecting $v_{\text {out }}=23.3 \mathrm{~V}$ or below would lead to instability of $K_{L M I-L Q R}$ ), the linear controller can reach the desired state even in the presence of parasitic elements. In this situation the linear controller, with a settling time of around $2 \mathrm{~ms}$, is faster than the proposed adaptive approach. But a final question arises: to which extent can the linear and the adaptive designs tolerate a change in the working point (duty cycle)? Robustness to variations of duty cycle was studied, e.g. in [16]: by changing the load, one necessarily requires to settle to a different duty cycle, which introduces uncertainty in the (averaged) model of the boost converter and might lead to instability. Fig. 7 shows that the linear controller can tolerate a $50 \%$ load variation, applied at time $0.02 \mathrm{~s}$. But, from Fig. 8 , we see that the linear controller cannot handle a $80 \%$ load variation: on the other hand, the adaptive controller can recover in both cases.

Therefore, we verified that, while the non-adaptive design needs an accurate model and its region of stability shrinks in the presence of duty cycle uncertainty, the adaptive design will estimate the actual load variation and compensate for the (possibly large) change of duty cycle. This is done, of course, at the expense of some learning transient, highlighted in Fig. 7 and Fig. 8. Therefore, the advantage of adaptive control is not in terms of performance (we have seen that the linear controller $K_{L M I-L Q R}$ might have a better settling time), but rather in providing improved stability in the presence of large uncertainty (may it be parametric uncertainty or changes of working point).

\section{Conclusions}

In this work we proposed an adaptive switching strategy for the design of pulse width modulation signals in power converters. We illustrated that a switched (i.e. hybrid) model better represents the power converter dynamics especially under lower frequency pulse width modulation. The uncertainties in the power converters parameters have been handled via an adaptive control approach. After defining the pulse width modulation in terms of a reverse mode-dependent dwell time, a desired level of performance was guaranteed by solving online a set of linear matrix inequalities. The LMIs are based on global uniformly ultimately boundedness of switched system under mode-dependent dwell time, and since the parameters of 

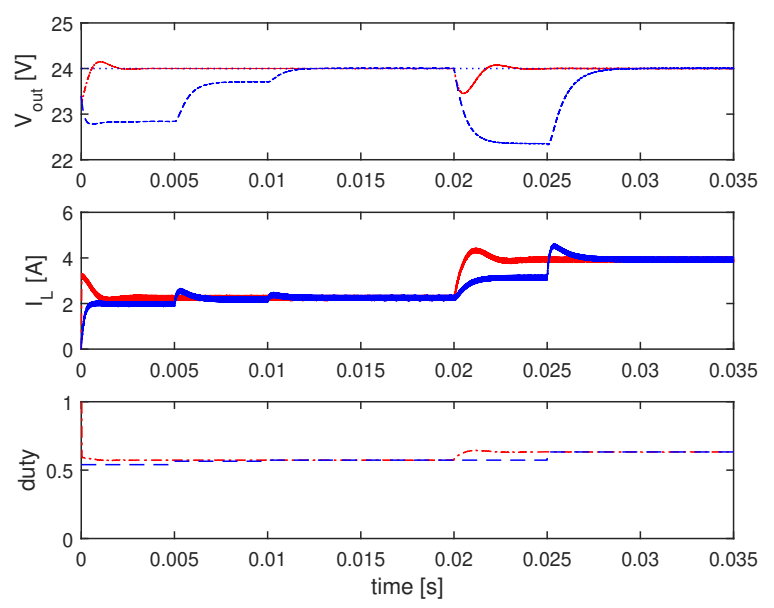

Figure 7: Non-ideal (lossy) boost converter with initial condition $v_{\text {out }}=23.4 \mathrm{~V}$ and $i_{L}=0 \mathrm{~A}$, and with $50 \%$ load variation at time 0.02s: Output voltage, inductor current and duty cycle for the linear controller (dash-dotted line), and the proposed adaptive strategy (dashed line). The desired voltage is indicated with a dotted line.

the power converter are uncertain, they are coupled with a parameter estimator and solved online in an adaptive fashion using an exploitation-exploration mechanism. The effectiveness of the proposed approach has been verified via simulations on boost converter: comparisons with a state-space design based on averaged model have been provided.

\section{Appendix A. Switched model of a boost converter}

Here we will show how to derive the switched affine model of a boost power converter. A DC-DC boost converter generates at steady state an average output voltage that is larger than its input voltage (Fig. A.9). By applying the Kirchhoff's circuit laws one obtains, for each mode, the following models: for the closed switch $(\sigma(t)=1)$

$$
\begin{aligned}
& A_{1}=\left[\begin{array}{cc}
-\left(\frac{R_{S 1}}{\frac{R_{S 1}}{R_{D 0}}+1}+R_{L}\right) \frac{1}{L} & \left(\frac{1}{\frac{R_{S 1}}{R_{D 0}}+1}-1\right) \frac{1}{L} \\
\left(\frac{1}{\frac{R_{D 0}}{R_{S 1}}+1}\right) \frac{1}{C} & -\left(\frac{1}{R_{S 1}+R_{D 0}}+\frac{1}{R_{\text {load }}}\right) \frac{1}{C}
\end{array}\right], \\
& E_{1}=\left[\begin{array}{c}
\frac{1}{L} \\
0
\end{array}\right] .
\end{aligned}
$$

For the open switch $(\sigma(t)=0)$

$$
\begin{aligned}
& A_{0}=\left[\begin{array}{cc}
-\left(\frac{R_{D 1}}{\frac{R_{S 0}}{R_{D 1}}+1}+R_{L}\right) \frac{1}{L} & \left(\frac{1}{\frac{R_{S 0}}{R_{D 1}}+1}-1\right) \frac{1}{L} \\
\left(\frac{1}{\frac{R_{D 1}}{R_{S 0}}+1}\right) \frac{1}{C} & -\left(\frac{1}{R_{S 0}+R_{D 1}}+\frac{1}{R_{\text {load }}}\right) \frac{1}{C}
\end{array}\right], \\
& E_{0}=\left[\begin{array}{c}
\frac{1}{L} \\
0
\end{array}\right] .
\end{aligned}
$$



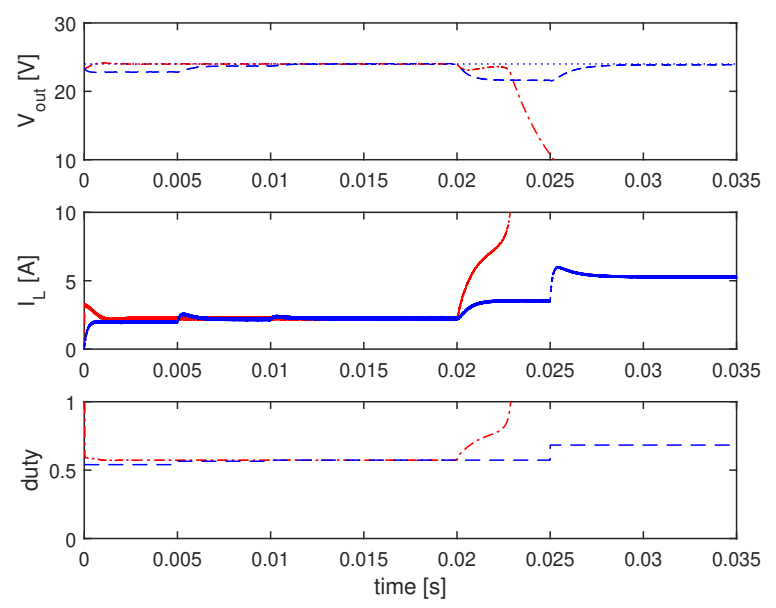

Figure 8: Non-ideal (lossy) boost converter with initial condition $v_{\text {out }}=23.4 \mathrm{~V}$ and $i_{L}=0 \mathrm{~A}$, and with $80 \%$ load variation at time 0.02s: Output voltage, inductor current and duty cycle for the linear controller (dash-dotted line), and the proposed adaptive strategy (dashed line). The desired voltage is indicated with a dotted line.

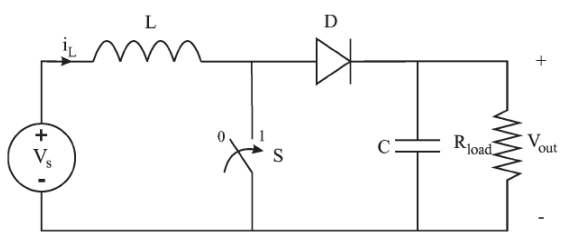

Figure A.9: Boost converter

The switch, inductance and diode can be modeled as nonideal lossy elements: in (A.1)-(A.2) $R_{S 0}$ and $R_{S 1}$ represent the losses of the switch, $C$ is the capacitance, $L$ is the inductance ( $R_{L}$ representing losses), $D$ is the diode ( $R_{D 0}$ and $R_{D 1}$ representing losses). Modeling the parasitic elements as open circuit resistance of the switches and inductance/diode resistances has been mutuated from [21]: there are alternative ways to model losses, and in particular switching losses, which have not been considered in this work.

Applying a similar approach to the other DC-DC converter configurations [1,2], analogous switched affine models can be obtained for buck or buck-boost converters. For completeness, we also give the averaged model of a power converter, not adopted in this work for control design but used in Figs. 1(c), 1(d) and 2. The model is derived from averaging theory using the following equation [16]

$$
\begin{aligned}
\dot{\tilde{x}}(t)= & \left(A_{0}+\left(A_{1}-A_{0}\right) D\right) \tilde{x}(t)+\left(A_{1}-A_{0}\right) \tilde{x}(t) \tilde{u}(t) \\
& +\left(\left(A_{1}-A_{0}\right) X+\left(E_{1}-E_{0}\right)\right) \tilde{u}(t) .
\end{aligned}
$$

The vectors $\tilde{x} \in \mathbb{R}^{2}$ and $\tilde{u} \in \mathbb{R}$ represent the incremental state and input respectively, while $X$ and $D$ are the equilibrium state and input (duty cycle) defining the nominal operating point.

In order to obtain a linear-in-the-parameters model, the switched model of the power converter is reparametrized. Due to the presence of matrix entries common to both modes, a convenient reparametriza- 
tion for the boost converter (A.1)-(A.2) is

$$
\begin{gathered}
A_{1}=\left[\begin{array}{cc}
-a_{1} & -a_{2} \\
a_{3} & -a_{4}
\end{array}\right], \quad A_{0}=\left[\begin{array}{cc}
-a_{5} & -a_{6} \\
a_{7} & -a_{8}
\end{array}\right] \\
E_{1}=E_{0}=\left[\begin{array}{c}
e_{1} \\
0
\end{array}\right]
\end{gathered}
$$

with 9 positive parameters to be estimated.

\section{Appendix B. Proof of Lemma 1}

The proof follows the lines of [32], with the peculiarity that a mode-dependent switching and global uniformly ultimately boundedness are considered. Denote the switching instants with $t_{k}, k \in \mathbb{N}_{+}$, and by convention in switched systems, we assume all functions to be continuous from right, i.e. $V\left(t_{k}^{+}\right)=$ $V\left(t_{k}\right)$. From the second inequality in (3) it can be seen that for all $t \in\left[t_{k}, t_{k+1}\right)$ the time derivative of the Lyapunov function $V\left(t, x(t)-x_{d e s}\right)=W_{\sigma(t)}\left(t, x(t)-x_{d e s}\right)$ along a trajectory of the switched system satisfies, $t \in\left[t_{k}, t_{k+1}\right)$

$$
V(t) \leq e^{-\lambda_{\sigma\left(t_{k}\right)}\left(t-t_{k}\right)} V\left(t_{k}\right)+c .
$$

Then, supposing system (1) had switched from mode $p$ to mode $q$ at time $t_{k}$, and switches from mode $q$ to mode $p$ at switching instant $t_{k+1}$ (known switching sequence as in [38]), we have

$$
V\left(t_{k+1}\right) \leq \mu_{\sigma\left(t_{k}\right)} \mu_{\sigma\left(t_{k-1}\right)} e^{-\lambda_{\sigma\left(t_{k}\right)}\left(t_{k+1}-t_{k}\right)-\lambda_{\sigma\left(t_{k-1}\right)}\left(t_{k}-t_{k-1}\right)} V\left(t_{k-1}\right)+c .
$$

Using the condition $\ln \mu_{1}+\ln \mu_{0}+\lambda_{1} T_{1}+\lambda_{0} T_{0}<0$, we have $\mu_{\sigma\left(t_{k}\right)} \mu_{\sigma\left(t_{k-1}\right)} e^{-\lambda_{\sigma\left(t_{k}\right)}\left(t_{k+1}-t_{k}\right)-\lambda_{\sigma\left(t_{k-1}\right)}\left(t_{k}-t_{k-1}\right)}<1$. We conclude that there exists a ball $\mathscr{B}(r)$ around the desired state, for some positive constant $r$ depending on $c_{p}$, and $\mu \in(0,1)$ such that

$$
V\left(x\left(t_{k}\right)\right) \leq \mu^{k} V\left(x_{0}\right), \quad \forall x \notin \mathscr{B}(r),
$$

which together with the fact that $V\left(x_{d e s}\right)=0$ implies that $x_{\text {des }}$ is GUUB. Using Lyapunov arguments [39, Thm. 4.18], the ball inside which the trajectory will remain can be calculated as follows. In between two consecutive switches the Lyapunov function decays at an exponential rate outside the following ball

$$
\left\|x-x_{\text {des }}\right\| \geq b_{c}
$$

where $b_{c}$ is such that

$$
\left\{x:\left\|x-x_{\text {des }}\right\| \leq b_{c}\right\} \subseteq\left\{x: V\left(x-x_{\text {des }}\right) \leq c\right\}:=\Omega_{c}
$$

To calculate the ultimate bound we see that

$$
x \in \Omega_{c} \Rightarrow\left\|x-x_{\text {des }}\right\| \leq \underline{a}^{-1}\left(\bar{a}\left(b_{c}\right)\right):=\varepsilon
$$

\section{Appendix C. Proof of Theorem 3}

We start by observing that according to Lemma 1 in [40], Lemma 1 in this manuscript implies that, for a switching sequence $\left\{t_{0}, t_{1}, \ldots, t_{k}, t_{k+1}, \ldots\right\}$ satisfying the reverse mode-dependent dwell time condition, there exists a family of continuously differentiable, positive definite, radially unbounded Lyapunov 
functions $\bar{W}_{\sigma}: \mathbb{R}^{n} \rightarrow \mathbb{R}_{+}$, positive constants $\varepsilon_{1}, \varepsilon_{2}, \varepsilon_{3}, \lambda$ and $0<\kappa<1$ which satisfy

$$
\begin{aligned}
& \left|\frac{\partial \bar{W}_{\sigma(t)}}{\partial x} E_{\sigma(t)}\right| \leq \varepsilon_{1} \text { and }\left\|x-x_{d e s}\right\| \geq \varepsilon_{3} \Rightarrow \frac{\partial \bar{W}_{\sigma(t)}}{\partial x}\left(A_{\sigma(t)} x(t)+E_{\sigma(t)} v_{s}\right)-\lambda \bar{W}_{\sigma(t)}<-\varepsilon_{2} \\
& \text { and } \bar{W}_{\sigma\left(t_{k+1}\right)}\left(x\left(t_{k+1}\right)\right) \leq \kappa \bar{W}_{\sigma\left(t_{k}\right)}\left(x\left(t_{k}\right)\right)
\end{aligned}
$$

Now, let us assume that (C.1) is verified for the multiple Lyapunov function arising from the solution of (9), with the actual system matrices of the power converter. Let us define the following region

$$
\mathscr{U}=\left\{x \in \mathbb{R}^{n} \mid\left\|x-x_{\text {des }}\right\| \geq \varepsilon_{3} \text { and }\left|\frac{\partial \bar{W}_{\sigma(t)}}{\partial x} E_{\sigma(t)}\right| \leq \varepsilon_{1}\right\}
$$

which is the region where, without any external excitation, the trajectories are converging towards $x_{d e s}$ (according to (C.1)).

Let us consider, for $\left\|x-x_{d e s}\right\|>\varepsilon_{3}$ and in between switches $t \in\left[t_{k}, t_{k+1}\right)$, the following cases:

C1) $x(t) \notin \mathscr{U}$ and $\left|\frac{\partial V_{\sigma\left(t_{k}\right)}}{\partial x} \hat{E}_{\sigma\left(t_{k}\right)}\right| \geq \eta$

Let

$$
\dot{\bar{W}}_{( \pm j)}(t)=\min _{j \in\{1, \ldots, m\}} \frac{\partial \bar{W}_{\sigma\left(t_{k}\right)}}{\partial x}\left[A_{\sigma\left(t_{k}\right)} x(t)+E_{\sigma\left(t_{k}\right)}\left(v_{s} \pm v_{s}^{(j)}\right)\right]-\lambda \bar{W}_{\sigma(t)},
$$

where $\pm v_{s}^{(j)}$ contains the perturbed input voltage as defined in (12). In the following we will omit the time index for simplicity. Since $\left|\frac{\partial \bar{W}_{\sigma\left(t_{k}\right)}}{\partial x} \hat{E}_{\sigma\left(t_{k}\right)}\right| \geq \eta$, we can verify that

$$
\min _{j \in\{1, \ldots, m\}} \frac{\partial \bar{W}_{\sigma\left(t_{k}\right)}}{\partial x} E_{\sigma\left(t_{k}\right)}\left[v_{s} \pm v_{s}^{(j)}\right]<-c \varepsilon_{1} \alpha
$$

for some positive constant $c$ independent of $\varepsilon_{1}$, and

$$
\min _{j \in\{1, \ldots, m\}} \dot{\bar{W}}_{( \pm j)}=\frac{\partial \bar{W}_{\sigma\left(t_{k}\right)}}{\partial x} \Theta_{\sigma\left(t_{k}\right)}^{*} \bar{x}(t)-c \varepsilon_{1} \alpha
$$

Now denote with $V_{\sigma\left(t_{k}\right)}$ the multiple Lyapunov function arising from (10) with the estimated matrices. By making the difference between the derivative of $\bar{W}_{\sigma\left(t_{k}\right)}$ along the trajectories of the real system and the derivative of $V_{\sigma\left(t_{k}\right)}$ along the trajectories of the estimated one, we obtain

$$
\begin{aligned}
\dot{\bar{W}}_{( \pm j)}-\dot{V}_{( \pm j)}= & \frac{\partial \bar{W}_{\sigma\left(t_{k}\right)}}{\partial x}\left[\Theta_{\sigma\left(t_{k}\right)}^{*} \bar{x}(t)+E_{\sigma\left(t_{k}\right)}\left( \pm v_{s}^{(j)}\right)\right]-\lambda \bar{W}_{\sigma(t)} \\
& -\frac{\partial V_{\sigma\left(t_{k}\right)}}{\partial x}\left[\hat{\Theta}_{\sigma\left(t_{k}\right)}^{*} \bar{x}(t)+\hat{E}_{\sigma\left(t_{k}\right)}\left( \pm v_{s}^{(j)}\right)\right]-\lambda V_{\sigma(t)} \\
= & \mathscr{O}(\|\tilde{\Theta}\|)+\mathscr{O}(\|\tilde{\Theta}\|)\left( \pm v_{s}^{(j)}\right)
\end{aligned}
$$

where $\bar{x}=\left[\begin{array}{ll}x^{\prime} & v_{s}^{\prime}\end{array}\right]^{\prime}$ and $\tilde{\Theta}_{\sigma\left(t_{k}\right)}=\Theta_{\sigma\left(t_{k}\right)}^{*}-\hat{\Theta}_{\sigma\left(t_{k}\right)}$. The persistency of excitation condition on the input voltage implies

$$
\tilde{\Theta}_{\sigma\left(t_{k}\right)}=\mathscr{O}\left(\frac{1}{k \gamma}\right),
$$


where $1 / k$ arises from the the total time when subsystem $\sigma\left(t_{k}\right)$ has been active (which is proportional to $k$ ). Using (C.5) and (C.6) we then obtain

$$
\dot{\bar{W}}_{( \pm j)}-\dot{V}_{( \pm j)}=\mathscr{O}\left(\frac{1}{k \gamma}\right)+\mathscr{O}\left(\frac{1}{k \gamma}\right) \alpha .
$$

Combining (C.7) and (C.4) we then obtain that, if

$$
\varepsilon_{1}>\mathscr{O}\left(\frac{1}{k \gamma}\right),
$$

then

$$
\arg \min _{j \in\{1, \ldots, m\}} \dot{\bar{W}}_{( \pm j)}=\arg \min _{j \in\{1, \ldots, m\}} \dot{V}_{( \pm j)}
$$

and $\exists \beta_{1}>0: \alpha_{k}>\beta_{1}$ we have $\dot{\bar{W}}(t)<-\lambda \bar{W}_{\sigma(t)}-\bar{\alpha}$, where $\bar{\alpha}$ is a positive constant.

C2) $x(t) \in \mathscr{U}$ and $\left|\frac{\partial V_{\sigma\left(t_{k}\right)}}{\partial x} \hat{E}_{\sigma\left(t_{k}\right)}\right|<\eta$

Using (C.1) we obtain

$$
\dot{\bar{W}}(t)<-\lambda \bar{W}_{\sigma(t)}-\varepsilon_{2}+\sqrt{m} \varepsilon_{1} \alpha
$$

which results in $\dot{W}(t)<-\lambda \bar{W}_{\sigma(t)}-\bar{\alpha}$ provided that $\alpha<\left(-\bar{\alpha}+\varepsilon_{2}\right) / \sqrt{m} \varepsilon_{1}=\beta_{2}$.

C3) $x(t) \notin \mathscr{U}$ and $\left|\frac{\partial V_{\sigma\left(t_{k}\right)}}{\partial x} \hat{E}_{\sigma\left(t_{k}\right)}\right|<\eta$

In this case we obtain

$$
\left|\frac{\partial \bar{W}_{\sigma\left(t_{k}\right)}}{\partial x} E_{\sigma\left(t_{k}\right)}-\frac{\partial V_{\sigma\left(t_{k}\right)}}{\partial x} \hat{E}_{\sigma\left(t_{k}\right)}\right| \leq \mathscr{O}\left(\frac{1}{k \gamma}\right)
$$

which implies that if $\mathscr{O}\left(\frac{1}{k \gamma}\right) \leq \varepsilon_{1}$ this case is never verified.

C4) $x(t) \notin \mathscr{U}$ and $\left|\frac{\partial V_{\sigma\left(t_{k}\right)}}{\partial x} \hat{E}_{\sigma\left(t_{k}\right)}\right| \geq \eta$

In this case we obtain that if $\mathscr{O}\left(\frac{1}{k \gamma}\right) \leq \varepsilon_{1}$ and

$$
\frac{1}{4} \varepsilon_{1} \leq \eta \leq \frac{1}{2} \varepsilon_{1}
$$

then

$$
\left|\frac{\partial \bar{W}_{\sigma\left(t_{k}\right)}}{\partial x} \hat{E}_{\sigma\left(t_{k}\right)}\right| \geq \frac{1}{4} \varepsilon_{1}
$$

which we can use to repeat the same reasoning after (C.3), provided that $c$ is replaced with $c / 4$. 
We have obtained that, in between switches, for $\left\|x-x_{d e s}\right\|>\varepsilon_{3}$ and for $\mathscr{O}\left(\frac{1}{k \gamma}\right)<\varepsilon_{1}$, the derivative of the Lyapunov function $\bar{W}$ might increase with bounded rate.

At consecutive switching times, (C.1) and the fact that due to convexity of the LMIs we have $\bar{W}_{\sigma\left(t_{k}\right)}-$ $V_{\sigma\left(t_{k}\right)}=\mathscr{O}(\|\tilde{\Theta}\|)$ give us

$$
\bar{W}_{\sigma\left(t_{k}^{+}\right)}\left(x\left(t_{k}\right)\right) \leq \kappa \bar{W}_{\sigma\left(t_{k}\right)}\left(x\left(t_{k}\right)\right)+\mathscr{O}\left(e^{-\frac{1}{k \gamma}}\right)
$$

which implies that there is a ball outside which the bounded increase in between switches are compensated by the decrease at jumps. Furthermore, this ball converges to $\left\|x-x_{\text {des }}\right\|>\varepsilon_{3}$ exponentially fast. By looking at the overall behavior of the Lyapunov function $\bar{W}$ we have that $\mathscr{O}\left(\frac{1}{k \gamma}\right) \geq \varepsilon_{1}$ in an interval that can be made as small as desired by increasing $\gamma$. After that, we have $\mathscr{O}\left(\frac{1}{k \gamma}\right)<\varepsilon_{1}$ and in view of C1)-C4) destabilizing effect for $\left\|x-x_{\text {des }}\right\| \geq \varepsilon_{3}$. Therefore, using invariant set theory, it is then possible to show closed-loop signal boundedness and convergence of $x(t)$ to the subset $\left\{x \in \mathbb{R}^{n}:\left\|x-x_{\text {des }}\right\|<\varepsilon_{3}\right\}$.

A final comment concerns the relation between the optimal $\varepsilon$ which can be achieve if the system matrices where perfectly known, and the actual bound $\varepsilon_{3}$. It is clear that $\varepsilon_{3}=\varepsilon$ only if $\varepsilon_{1}=\varepsilon_{1}^{*}$ and $\varepsilon_{2}=\varepsilon_{2}^{*}$ where $\varepsilon_{1}^{*}$ and $\varepsilon_{2}^{*}$ represent the true bounds assuming the system matrices are known: obviously such bounds are in general unknown a priori, therefore the choice of $\eta$, which should satisfy (C.12) and the choice of $\beta_{1}, \beta_{2}$ which affect $\bar{\alpha}$ together with $\varepsilon_{2}$, require some fine tuning. This is often the case in adaptive control designs, where closed-loop stability is guaranteed provided that the control design constants satisfy certain bounds that in general are difficult to be calculated a priori [40, 41].

\section{References}

\section{References}

[1] S. Sangwine. Electronic Components and Technology, Third Edition. CRC Press, 2007.

[2] R. W. Erickson and D. Maksimovic. Fundamentals of Power Electronics, Third Edition. Springer US, 2001.

[3] R. D. Middlebrook and S. Cuk. A general unified approach to modelling switching-converter power stages. In 1976 IEEE Power Electronics Specialists Conference, pages 18-34, 1976.

[4] G. Escobar, A. J. van der Schaft, and R. Ortega. A Hamiltonian viewpoint in the modelling of switching power converters. Automatica, 35:445-452, 1999.

[5] L. Iannelli, K. H. Johansson, U. T. Jonsson, and F. Vasca. Subtleties in the averaging of a class of hybrid systems with applications to power converters. Control Engineering Practice, 16:961-975, 2008.

[6] J. C. Geromel and P. Colaneri. Stability and stabilization of continuous-time switched linear systems. SIAM Journal on Control and Optimization, 45:1915-1930, 2006.

[7] D. Liberzon. Switching in Systems and Control. Birkhauser, Boston, MA, 2003.

[8] L. I. Allerhand and U. Shaked. Robust stability and stabilization of linear switched systems with dwell time. IEEE Transactions on Automatic Control, 56(2):381-386, 2011.

[9] W. Xiang. On equivalence of two stability criteria for continuous-time switched systems with dwell time constraint. Automatica, 54:36-40, 2015.

[10] S. Yuan, B. De Schutter, and S. Baldi. Adaptive asymptotic tracking control of uncertain time-driven switched linear systems. IEEE Transactions on Automatic Control, 62(11):5802-5807, 2017.

[11] S. Yuan, L. Zhang, B. De Schutter, and S. Baldi. A novel lyapunov function for a non-weighted 12 gain of asynchronously switched linear systems. Automatica, 87:310 - 317, 2018.

[12] G. S. Deaecto, J. C. Geromel, F. S. Garcia, and J. A. Pomilio. Switched affine systems control design with application to DC-DC converters. IET Control Theory Applications, 4(7):1201-1210, 2010.

[13] J. Buisson, P.-Y. Richard, and H. Cormerais. On the Stabilisation of Switching Electrical Power Converters, pages 184-197. Springer Berlin Heidelberg, Berlin, Heidelberg, 2005.

[14] M. Rubensson and B. Lennartson. Global convergence analysis for piecewise linear systems applied to limit cycles in a DC/DC converter. In Proceedings of the 2002 American Control Conference, volume 2, pages 1272-1277, 2002. 
[15] C. Albea, G. Garcia, and L. Zaccarian. Hybrid dynamic modeling and control of switched affine systems: Application to DC-DC converters. In 2015 54th IEEE Conference on Decision and Control (CDC), pages 2264-2269, 2015.

[16] C. Olalla, R. Leyva, A. El Aroudi, and I. Queinnec. Robust LQR control for PWM converters: An LMI approach. IEEE Transactions on Industrial Electronics, 56:2548-2558, 2009.

[17] F. Amato, C. Cosentino, A. S. Fiorillo, and A. Merola. Stabilization of bilinear systems via linear state-feedback control. IEEE Transactions on Circuits and Systems II: Express Briefs, 56(1):76-80, 2009.

[18] C. Olalla, R. Leyva, A. El Aroudi, P. Garces, and I. Queinnec. LMI robust control design for boost PWM converters. IET Power Electronics, 3:75-85, 2010.

[19] C. Olalla, I. Queinnec, R. Leyva, and A. El Aroudi. Optimal state-feedback control of bilinear DC-DC converters with guaranteed regions of stability. IEEE Transactions on Industrial Electronics, 59:3868-3880, 2012.

[20] N. Athanasopoulos, A. I. Doban, and M. Lazar. Constrained stabilization of discrete-time linear systems. 21 st Mediterranean Conference on Control \& Automation (MED), 25-28 June, pages 830-839, 2013.

[21] F. M. Oettmeier, J. Neely, S. Pekarek, R. DeCarlo, and K. Uthaichana. MPC of switching in a boost converter using a hybrid state model with a sliding mode observer. IEEE Transactions on Industrial Electronics, 56:3453-3466, 2009.

[22] A. Salvi, S. Santini, D. Biel, J. M. Olm, and M. di Bernardo. Model reference adaptive control of a full-bridge buck inverter with minimal controller synthesis. 52nd IEEE Conference on Decision and Control December 10-13, Florence, Italy, pages 3469-3474, 2013.

[23] R.-J. Wai, M.-W. Chen, and Y.-K. Liu. Design of adaptive control and fuzzy neural network control for single-stage boost inverter. IEEE Transactions on Industrial Electronics, 62:5434-5445, 2015.

[24] C. Albea, F. Gordillo, and C. Canudas-de Wit. Adaptive control design for a boost inverter. Control Engineering Practice, 19:32-44, 2011.

[25] H. El-Fadil and F. Giri. Robust nonlinear adaptive control of multiphase synchronous buck power converters. Control Engineering Practice, 17:1245-1254, 2009.

[26] Y. Abdel-Rady, I. Mohamed, and E. F. El-Saadany. An improved deadbeat current control scheme with a novel adaptive selftuning load model for a three-phase PWM voltage-source inverter. IEEE Transactions on Industrial Electronics, 54:747-759, 2007.

[27] T. D. Do, V. Q. Leu, Y.-S. Choi, H. H. Choi, and J.-W. Jung. An adaptive voltage control strategy of three-phase inverter for stand-alone distributed generation systems. IEEE Transactions on Industrial Electronics, 60:5660-5672, 2013.

[28] L. Corradini, A. Costabeber, P. Mattavelli, and S. Saggini. Parameter-independent time-optimal digital control for point-ofload converters. IEEE Transactions on Power Electronics, 24(10):2235-2248, 2009.

[29] J. P. Hespanha, D. Liberzon, and A. R. Teel. On input-to-state stability of impulsive systems. In Proceedings of the 44th IEEE Conference on Decision and Control, pages 3992-3997, 2005.

[30] X. Zhao, L. Zhang, P. Shi, and M. Liu. Stability and stabilization of switched linear systems with mode-dependent average dwell time. IEEE Transactions on Automatic Control, 57:1809-1815, 2012.

[31] E. Lavretsky and K. Wise. Robust and Adaptive Control: With Aerospace Applications. Springer, 2013.

[32] W. Xiang and J. Xiao. Stabilization of switched continuous-time systems with all modes unstable via dwell time switching. Automatica, 50(3):940 - 945, 2014.

[33] P. A. Ioannou and J. Sun. Robust Adaptive Control. Dover Publications, 2012.

[34] R. De Keyser, J. Bonilla, and I. Ionescu. A comparative study of several control techniques applied to a boost converter. Proceeding IEEE 10th International Conference on Optimization of Electrical and Electronic Equipment (OPTIM), Brasov, Romania, pages 71-78, 2006.

[35] L. Zhang and W. Xiang. Mode-identifying time estimation and switching-delay tolerant control for switched systems: An elementary time unit approach. Automatica, 64:174-181, 2016.

[36] Y. Zhang, H. Wang, B. Li, D. Xu, and F. Blaabjerg. Precharge strategies for isolated modular DC-DC converters under two different start-up conditions. In 2017 IEEE 26th International Symposium on Industrial Electronics (ISIE), pages 1011-1016, 2017.

[37] A. Domahidi, E. Chu, and S. Boyd. ECOS: An SOCP solver for embedded systems. In 2013 European Control Conference (ECC), pages 3071-3076, 2013.

[38] S. Yuan, B. De Schutter, and S. Baldi. Robust adaptive tracking control of uncertain slowly switched linear systems. Nonlinear Analysis: Hybrid Systems, 27:1 - 12, 2018.

[39] H. K. Khalil. Nonlinear Systems. New York: Macmillan, 1992.

[40] E. B. Kosmatopoulos and P. A. Ioannou. Robust switching adaptive control of multi-input nonlinear systems. IEEE Transactions on Automatic Control, 47(4):610-624, 2002.

[41] E. B. Kosmatopoulos. An adaptive optimization scheme with satisfactory transient performance. Automatica, $45(3): 716$ 723, 2009. 